PROCEEDINGS OF THE

AMERICAN MATHEMATICAL SOCIETY

Volume 128, Number 4, Pages 943-951

S 0002-9939(99)05045-5

Article electronically published on July 28, 1999

\title{
ON THE GELFAND-KIRILLOV CONJECTURE FOR QUANTUM ALGEBRAS
}

\author{
PHILIPPE CALDERO
}

(Communicated by Roe Goodman)

\begin{abstract}
Let $q$ be a complex not a root of unity and $\mathfrak{g}$ be a semi-simple Lie $\mathbb{C}$-algebra. Let $U_{q}(\mathfrak{g})$ be the quantized enveloping algebra of Drinfeld and Jimbo, $U_{q}\left(\mathfrak{n}^{-}\right) \otimes U^{0} \otimes U_{q}(\mathfrak{n})$ be its triangular decomposition, and $\mathbb{C}_{q}[G]$ the associated quantum group. We describe explicitly Fract $U_{q}(\mathfrak{n})$ and Fract $\mathbb{C}_{q}[G]$ as a quantum Weyl field. We use for this a quantum analogue of the Taylor lemma.
\end{abstract}

\section{INTRODUCTION}

Let $q$ be a nonzero complex number which is not a root of unity. In this article, a $\mathbb{C}$-algebra defined by generators $X_{i}, 1 \leq i \leq m$, and relations $X_{i} X_{j}=q^{a_{i, j}} X_{j} X_{i}$, $1 \leq i<j \leq m, a_{i, j} \in \mathbb{Z}$, will be called "the algebra of regular functions on an affine quantum space". Its skew field of fractions will be called the quantum Weyl field. The $X_{i}, 1 \leq i \leq m$, will be called a system of $q$-commuting generators (SQCG).

Let $\mathfrak{g}$ be a semi-simple Lie $\mathbb{C}$-algebra of rank $n$. Let $R$ be the root system associated to the choice of a Cartan subalgebra $\mathfrak{h}$. We denote by $\Delta=\left\{\alpha_{i}\right\}$ the set of simple roots of $R, P$ the lattice of associated weights generated by the fundamental weights $\varpi_{i}, 1 \leq i \leq n$, and $P^{+}:=\sum_{i} \mathbb{N} \varpi_{i}$ the lattice of dominant weights. Let $G$ be the simply connected group associated to $\mathfrak{g}$ and $U_{q}(\mathfrak{g})$ the Drinfeld and Jimbo's quantized enveloping algebra. We define as in the classical case its "nilpotent" subalgebra $U_{q}(\mathfrak{n})$ and the quantum algebra of regular functions on the group $\mathbb{C}_{q}[G]$. A theorem of J. Alev and F. Dumas (cf. [1]) asserts that Fract $U_{q}(\mathfrak{n})$ is a quantum Weyl field when $\mathfrak{g}$ is of type $A_{n}$. In [15], A. Joseph proves that this property is verified for all semi-simple Lie algebras $\mathfrak{g}$ when $q$ is generic. We prove in this article that Fract $U_{q}(\mathfrak{n})$ and Fract $\mathbb{C}_{q}[G]$ are quantum Weyl fields when $\mathfrak{g}$ is semi-simple and when $q$ is not a root of one; see [9] for the case where $q$ is a root of one. The method we used provides a system of $q$-commuting generators.

Inspired by [12, Theorem 3.2], we essentially used the quantum analogue of the Taylor lemma. This lemma asserts that if 1$) \delta$ is a locally nilpotent $\sigma$-derivation (cf. 1.1) on a $\mathbb{C}$-algebra $A$ and 2) there exists an element $a$ such that $\delta(a)=1$, then $a$ is (right) transcendant on the invariant algebra $A^{\delta}$ and $A \simeq A^{\delta}[a]$.

Our results are proved as follows:

Received by the editors March 27, 1997 and, in revised form, May 15, 1998.

1991 Mathematics Subject Classification. Primary 17Bxx.

Key words and phrases. Quantum groups, quantum Weyl fields, $R$-matrix.

(C)2000 American Mathematical Society 
As a first step, we give (cf. Proposition 2.1) a multi-parametered version of the Taylor lemma for the locally nilpotent action (as a bialgebra) of the Borel subalgebra $U_{q}(\mathfrak{b})$ on an algebra $A$. The difficulty encountered in the quantum case is the following : the generators $E_{\beta}$ ( $\beta$ being a positive root) of the Poincaré-BirkhoffWitt base of $U_{q}(\mathfrak{n})$ do not act as $\sigma$-derivations on $A$. To get round this problem, we can, from a reduced decomposition of the longest element $w_{0}$ in the Weyl group, define a total order on the set of these generators and obtain a decreasing sequence of subalgebras $U_{q}\left(\mathfrak{n}_{\beta}\right)$ of $U_{q}(\mathfrak{n})$; cf. [10, Lemma 1.7]. With the help of a result of S.Z. Levendorskii and Y.S Soibelman (cf. [17, 2.4.1]) we obtain that $E_{\beta}$ acts as a $\sigma$-derivation on the subalgebra of $U_{q}\left(\mathfrak{n}_{\beta}<\right)$-invariants of $A, \beta^{<}$being the root preceding $\beta$. So, we can inductively apply the Taylor lemma and prove Proposition 2.1 .

As a second step, we apply Proposition 2.1, see also Assertion 2.2, to the (right) regular action of $U_{q}(\mathfrak{b})$ on $\mathbb{C}_{q}[G]$. Recall (cf. 1.4) that $\mathbb{C}_{q}[G]$ is generated as a space by the coefficients $c_{\mu, \nu}^{\lambda}$ of the simple finite dimensional $U_{q}(\mathfrak{g})$-modules $L_{q}(\lambda)$, $\lambda \in P^{+}$. Let $w_{0}=s_{i_{1}} \ldots s_{i_{N}}$ be a reduced decomposition of $w_{0}$ into a product of elementary reflections. Let $\beta=\beta_{l}:=s_{i_{1}} \ldots s_{i_{l-1}}\left(\alpha_{i_{l}}\right)$ and $y_{l}=s_{i_{1}} \ldots s_{i_{l}}$. By using the Lusztig automorphisms and the Weyl character formula, we prove that $c_{\beta}:=c_{y_{l-1} \varpi_{i_{l}}, \varpi_{i_{l}}}^{\varpi_{i_{l}}}$,is $U_{q}\left(\mathfrak{n}_{\beta}<\right)$-invariant. Moreover, with the help of the $\mathcal{R}$-matrix, we prove (cf. Proposition 2.3) that the $c_{\beta} q$-commute, i.e. commute up to a power of $q$. By the quantized Taylor lemma and the Drinfeld duality, we obtain the claimed theorem for Fract $U_{q}(\mathfrak{n})$. We may specify the description of Fract $U_{q}(\mathfrak{n})$ as in [1 Théorème 2.15]; cf. Theorem 3.2. We give similar results for the quantum algebras $S_{w}^{+}$of regular functions on a Schubert variety; cf. [14, 10.3.1 (3)]. On this subject, we remark that the elements $c_{\beta}$ belong to the Lakshmibai-Reshetikhin base of standard monomials [16. After localization, they generate a polynomial base.

As a third step, we show that our method works for $\mathbb{C}_{q}[G]$. If $\rho$ is the sum of fundamental weights, then the elements $d_{\beta}=c_{y_{l-1} \rho,-y_{l} \rho}^{\rho}, d_{\beta}^{\prime}=c_{y_{l-1} \rho,-y_{l-1} \rho}^{\rho}$ and $c_{w_{0} \varpi_{i}, \varpi_{i}}^{\varpi_{i}}$ generate the quantum Weyl field Fract $\mathbb{C}_{q}[G]$. This theorem is a consequence of the Taylor lemma for the regular action of $U_{q}(\mathfrak{b}) \otimes U_{q}(\mathfrak{b})^{\text {opp }}$ on $\mathbb{C}_{q}[G]$. Note that this result was proved by A.N. Panov for $G=S L_{n}$ and generic $q$ 21 .

In the classical case, the Gelfand-Kirillov conjecture asks if the enveloping algebra of $\mathfrak{g}$ is a Weyl field. In [12, A. Joseph gives a generalization of the Gelfand-Kirillov conjecture, replacing the enveloping algebra of $\mathfrak{g}$ by an algebra on which $\mathfrak{n}$ acts by derivations. The title of our article must be understood in the sense of this generalization. At the present time, we do not know if Fract $U_{q}(\mathfrak{g})$ is a quantum Weyl field. As for the classical case, this assertion may be shown when $\mathfrak{g}$ has type $A_{n}$ (see [19]).

We are in debt to T. Levasseur for his remarks and observations.

\section{Preliminaries and notations}

1.1. Let $\mathfrak{g}$ be a semi-simple Lie $\mathbb{C}$-algebra of rank $n$. We fix a Cartan sub-algebra $\mathfrak{h}$ of $\mathfrak{g}$. Let $\mathfrak{g}=\mathfrak{n}^{-}+\mathfrak{h}+\mathfrak{n}$ be the triangular decomposition and $\left\{\alpha_{i}\right\}_{i}$ be a base of the root system $\Delta$ resulting from this decomposition. We note $\mathfrak{b}=\mathfrak{n}+\mathfrak{h}$ and $\mathfrak{b}^{-}=\mathfrak{n}^{-}+\mathfrak{h}$, the two opposed Borel sub-algebras. Let $P$ be the weight lattice generated by the fundamental weights $\varpi_{i}, 1 \leq i \leq n$, and $P^{+}:=\sum_{i} \mathbb{N}_{i}$ the 
semigroup of integral dominant weights. We denote by $\rho$ the sum of fundamental weights. Let $W$ be the Weyl group, generated by the reflections corresponding to the simple roots $s_{\alpha_{i}}$. Let $w_{0}$ be the longest element of $W$. We denote by $($,$) the$ $W$-invariant form on $P$. We have $\left(\alpha_{j}, \varpi_{i}\right)=\delta_{i j} \frac{\left(\alpha_{i}, \alpha_{i}\right)}{2}$.

1.2. Let $q$ be a nonzero complex number not a root of unity and $U_{q}(\mathfrak{g})$ be the simply connected quantized enveloping algebra, defined as in [14, 3.2.9]. Let $U_{q}(\mathfrak{n})$, resp. $U_{q}\left(\mathfrak{n}^{-}\right)$, be the subalgebra generated by the canonical generators $E_{\alpha_{i}}$, resp. $F_{\alpha_{i}}$, of positive, resp. negative, weights. For all $\lambda$ in $P$, let $\tau(\lambda)$ be the corresponding element in the algebra $U^{0}$ of the torus of $U_{q}(\mathfrak{g})$. We have the triangular decomposition $U_{q}(\mathfrak{g})=U_{q}\left(\mathfrak{n}^{-}\right) \otimes U^{0} \otimes U_{q}(\mathfrak{n})$. We set

$$
U_{q}(\mathfrak{b})=U_{q}(\mathfrak{n}) \otimes U^{0}, \quad U_{q}\left(\mathfrak{b}^{-}\right)=U_{q}\left(\mathfrak{n}^{-}\right) \otimes U^{0} .
$$

$U_{q}(\mathfrak{g})$ is endowed with a structure of Hopf algebra with comultiplication $\Delta$, and antipode $S$.

We fix the following notations, where $t$ is a complex not root of one, $n$ a nonnegative integer and $\alpha$ a positive root : $[n]_{t}=\frac{1-q^{t}}{1-q},[n]_{t} !=[n]_{t}[n-1]_{t} \ldots[1]_{t}$, $q_{\alpha}=q^{\frac{(\alpha, \alpha)}{2}}$.

1.3. For $w$ in $W$, let $T_{w}$ be the Lusztig automorphism [18] associated to $w$. We fix a decomposition of the longest element of the Weyl group $w_{0}=s_{i_{1}} \ldots s_{i_{N}}$, where $N=\operatorname{dim} \mathfrak{n}$. This decomposition settles an order, denoted $<$, into the set $\Delta^{+}$of positive roots : $\beta_{N}=s_{i_{1}} \ldots s_{i_{N-1}}\left(\alpha_{i_{N}}\right), \ldots, \beta_{2}=s_{i_{1}}\left(\alpha_{i_{2}}\right), \beta_{1}=\alpha_{i_{1}}$. Then, we introduce the following elements in $U_{q}(\mathfrak{n}): E_{\beta_{s}}=T_{i_{1}} \ldots T_{i_{s-1}}\left(E_{i_{s}}\right)$. We define in the same way $F_{\beta_{s}}=T_{i_{1}} \ldots T_{i_{s-1}}\left(F_{i_{s}}\right)$.

We know (cf. [18) that these elements generate a Poincaré-Birkoff-Witt base of $U_{q}(\mathfrak{n})$. We have, by [22], see also [10, Lemma 1.7]:

Proposition. Let $U_{q}\left(\mathfrak{n}_{\beta}\right)$ be the space generated by the ordered products $\prod E_{\alpha}^{k_{\alpha}}$, $\alpha \in \Delta^{+}, \alpha \leq \beta, k_{\alpha} \in \mathbb{N}$. Then $U_{q}\left(\mathfrak{n}_{\beta}\right)$ is a subalgebra of $U_{q}(\mathfrak{n})$. Moreover, if $\mu<\beta$, we have $E_{\mu} E_{\beta}-q^{-(\mu, \beta)} E_{\beta} E_{\mu} \in \sum_{\alpha<\beta} U_{q}\left(\mathfrak{n}_{\beta}\right) E_{\alpha}$.

1.4. The dual $U_{q}(\mathfrak{g})^{*}$ is endowed with a structure of a left, resp. right, $U_{q}(\mathfrak{g})$ -

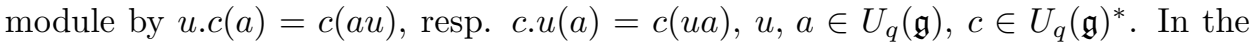
same way, if $M$ is a left $U_{q}(\mathfrak{g})$-module, we endow the dual $M^{*}$ with the structure of a right $U_{q}(\mathfrak{g})$-module by $\xi u(v)=\xi(u v), u \in U_{q}(\mathfrak{g}), \xi \in M^{*}, v \in M$.

For all $\lambda$ in $P^{+}$, let $L_{q}(\lambda)$ be the simple $U_{q}(\mathfrak{g})$-module with highest weight $\lambda$. We know that $L_{q}(\lambda)$ verifies the Weyl character formula, for all $w$ in $W$ we denote by $v_{w \lambda}$ the extremal vector of weight $w \lambda$. For all integral dominant weight $\lambda$, we fix a weight base $\left(v_{\mu}\right), \mu \in \Omega\left(L_{q}(\lambda)\right)$, of $L_{q}(\lambda)$. We denote by $\left(v_{\mu}^{*}\right)$ its dual base. From [14 10.2], we have the assertion

Assertion. Let $\lambda$ be an integral dominant weight and $w$ an element of the Weyl group. Fix a space $M$ and an isomorphism $\phi: M \rightarrow L_{q}(\lambda)^{*}$. We can endow $M$ with the structure of a right $U_{q}(\mathfrak{g})$-module by $: v^{*} . u=\phi^{-1}\left(\phi\left(v^{*}\right) T_{w}(u)\right), v^{*} \in M$. Then the $U_{q}(\mathfrak{g})$-module $M$ is isomorphic to $L_{q}(\lambda)^{*}$ and $\phi^{-1}\left(v_{w \lambda}^{*}\right)$, resp. $\phi^{-1}\left(v_{w w_{0} \lambda}^{*}\right)$, is its highest weight, resp. lowest weight, vector.

For all $\xi$ in $L_{q}(\lambda)^{*}$ and $v$ in $L_{q}(\lambda)$, let $c_{\xi, v}^{\lambda}$ in $U_{q}(\mathfrak{g})^{*}$ given by $c_{\xi, v}^{\lambda}(u)=\xi(u v)$, $u \in U_{q}(\mathfrak{g})$. Then we have $u . c_{\xi, v}^{\lambda}=c_{\xi, u v}^{\lambda}$ and $c_{\xi, v}^{\lambda} \cdot u=c_{\xi u, v}^{\lambda}$. If $\xi$, resp. $v$, has weight 
$\nu$, resp $\mu$, we set (if no confusion occurs) $c_{\nu, \mu}^{\lambda}=c_{\xi, v}^{\lambda}$. For all integral dominant weight $\lambda$, let $C(\lambda)$, resp. $C^{+}(\lambda)$, be the space generated by the $c_{\xi, v}^{\lambda}$, resp. $c_{\xi, \lambda}^{\lambda}$, $\xi \in L_{q}(\lambda)^{*}, v \in L_{q}(\lambda)$. We note $R=\mathbb{C}_{q}[G]=\bigoplus_{\lambda \in P^{+}} C(\lambda), R^{+}=\bigoplus_{\lambda \in P^{+}} C^{+}(\lambda)$. $R^{+}$and $R$ are subalgebras of the Hopf dual of $U_{q}(\mathfrak{g})$.

For $w$ in $W$, we define the quantized algebra $S_{w}^{+}$of regular functions on the Schubert variety (see [14, [15] for details) : $S_{w}^{+}$is the inductive limit of $\left(c_{w \lambda, \lambda}^{\lambda}\right)^{-1} V_{w}^{+}(\lambda)^{*}$, for $\lambda$ in $P^{+}$, where $V_{w}^{+}(\lambda)^{*}$ is the dual of the Demazure module $V_{w}(\lambda)$, naturally identified as a quotient of $C^{+}(\lambda)$.

1.5. We know that $U_{q}(\mathfrak{g})$ is an almost cocommutative Hopf algebra; cf. [11. Let $\mathcal{R}=\mathcal{R}_{(1)} \otimes \mathcal{R}_{(2)}$ be the $\mathcal{R}$-matrix of $U_{q}(\mathfrak{g})$. This satisfies $\mathcal{R} \Delta=\Delta^{t} \mathcal{R}$, where $t$ is the twist. From this property it follows easily that:

$$
\left(c \cdot \mathcal{R}_{(1)}\right)\left(c^{\prime} \cdot \mathcal{R}_{(2)}\right)=\left(\mathcal{R}_{(2)} \cdot c^{\prime}\right)\left(\mathcal{R}_{(1)} \cdot c\right), \quad c, c^{\prime} \in \mathbb{C}_{q}[G] .
$$

We recall the expression of the $\mathcal{R}$-matrix as an ordered product [17, 3.3]:

$$
\mathcal{R}=\left(\prod_{\alpha \in \Delta^{+}} \exp _{q_{\alpha}^{-2}}\left(\left(1-q_{\alpha}^{-2}\right) E_{\alpha} \otimes F_{\alpha}\right)\right) \tau(\gamma) \otimes \tau(\gamma),
$$

where $\gamma \in P, \exp _{t}(x)=\sum_{n \geq 0} \frac{x^{n}}{[n]_{t} !}$.

\section{A quantum TAYlor lemma}

2.1. We have the following lemma, whose proof is an analogue to [20, 1.1], [8, Proposition 1.1]:

Lemma. Let $A$ be an $\mathbb{C}$-algebra, $\sigma$ a $\mathbb{C}$-automorphism of $A, \delta$ a $\sigma$-derivation of $A$, i.e. $\delta(a b)=\delta(a) b+\sigma(a) \delta(b), a, b \in A$. Let $A^{\delta}$ be the algebra of $\delta$-invariants in A. Suppose that 1) $\delta$ is locally nilpotent, 2) $\sigma \delta \sigma^{-1}=Q \delta, Q \in \mathbb{C}^{*}, Q$ not root of one, 3) there exists $a$ in $A$ such that $\delta(a) \in \mathbb{C}^{*}$. Then $A=A^{\delta}[a]$ and $a$ is (right) transcendantal on $A^{\delta}$, i.e. $A=\bigoplus_{p \geq 0} A^{\delta} a^{p}$.

Proof. By 2), $A^{\delta}$ is $\sigma$-stable. Moreover, we have : $\delta^{p}\left(a^{p}\right)=[p]_{Q^{-1}} ! \delta(a)^{p}$. This implies the direct sum in the claimed equality. Let $u$ be in $A$, with degree $p$, i.e. $p$ is the greatest integer such that $u_{0}:=\delta^{p}(u) \neq 0$. Clearly, the element $u_{0}$ is in $A^{\delta}$. We prove the result by induction on $p$ by considering $u-\frac{1}{[p]_{Q^{-1} ! \delta(a)}} \sigma^{-p}\left(u_{0}\right) a^{p}$, of degree $\leq p-1$.

Let $A$ be an $\mathbb{C}$-algebra such that $U_{q}(\mathfrak{b})$ acts (as a bialgebra) on $A$, i.e. $A$ is a $U_{q}(\mathfrak{b})$-module and $a(u v)=a_{(1)} u a_{(2)} v, u, v \in A, a \in U_{q}(\mathfrak{b}), \Delta(a)=a_{(1)} \otimes a_{(2)}$. Suppose that this action is locally finite. We set $A^{0}=A$ and we note $A^{l}, 1 \leq l \leq N$, the algebra of $U_{q}\left(\mathfrak{n}_{\beta_{l}}\right)$-invariants in $A$. This proposition follows from the lemma.

Proposition. Let $A$ be an algebra defined as above. Suppose that, for all $\beta$ in $\Delta^{+}$, there exists $a_{\beta}$ in $A$ such that $E_{\alpha} \cdot a_{\beta}=\delta_{\alpha \beta}, \alpha \leq \beta$, where $\delta_{\alpha \beta}$ is the Kroenecker symbol.

Then, for all $l, 1 \leq l \leq N$, we have

$$
A=\bigoplus_{\left(k_{1}, \ldots, k_{l}\right) \in \mathbb{N}^{l}} A^{l} a_{\beta_{l}}^{k_{l}} \ldots a_{\beta_{1}}^{k_{1}} .
$$


Proof. We note $\phi: U_{q}(\mathfrak{b}) \rightarrow \operatorname{End}(A)$, the natural morphism for this action. By Proposition 1.3 and [17, 2.4.1], $\delta:=\phi\left(E_{\beta_{l}}\right)$ is a $\phi\left(\tau\left(\beta_{l}\right)\right)$-derivation on $A^{l-1}$. The conditions of the previous lemma are satisfied because 1) $\delta$ is locally nilpotent on $\left.A^{l-1}, 2\right) \tau\left(\beta_{l}\right)$ and $E_{\beta_{l}} q$-commute, 3) $a:=a_{\beta_{l}}$ is in $A^{l-1}$ and satisfies $\delta(a)=1$ by the definition. The proposition is obtained by induction on $l$ using the previous lemma.

2.2. We shall see that if $A$ is one of the algebras considered in the introduction, then the elements $a_{\beta}$ of Proposition 2.1 exist in some localization of $A$, and not in the algebra $A$. For the classical case, cf. [12, Theorem 2.6], it is enough to localize by a set $S$ generated by $\mathfrak{n}$-invariant elements in $A$. We can then apply the Taylor lemma to $A_{S}$. In the quantum case, the Taylor lemma needs some refinements. We slightly modify Lemma 2.1 to get

Assertion. Let $C$ be a noetherian domain on $\mathbb{C}, \sigma a \mathbb{C}$-automorphism of $C$ and $\delta$ be a $\sigma$-derivation on $C$ which satisfies 1) and 2) of Lemma 2.1. Suppose s (nonzero) and $s^{\prime}$ are $q$-commuting in $C$ and such that $\delta\left(s^{\prime}\right)=s \in C^{\delta}$. Let $a=s^{\prime} s^{-1}$ in Fract $C$ and $M=\bigcup_{p \geq 0} C s^{-p}$. Then $\delta$ acts on $M$ and (as spaces) $C \subset M=\bigoplus_{p \geq 0} M^{\delta} a^{p}$.

Proof. The element $s$ in $\delta$-invariant, so $\delta$ extends as a locally nilpotent derivation on $M$. Clearly, $C$ is a subset of $M$ and $\delta(a)=1$. The direct sum is proved as in Lemma 2.1. As $s$ and $s^{\prime} q$-commute, we have $\bigoplus_{p \geq 0} M^{\delta} a^{p} \subset M$. The reverse inclusion is an easy induction as in the proof of Lemma 2.1.

We now give a condition on $A$ which implies that Fract $A$ is isomorphic to a quantum Weyl field.

Definition. Let $A$ be a noetherian domain. We say that $A$ verifies the property $(\mathcal{P})$ if the following hypotheses are verified:

(i) $U_{q}(\mathfrak{b})$ acts (as a Hopf algebra) on the $\mathbb{C}$-algebra $A$ and this action is locally finite. Let $B=A^{N}$ be the subalgebra of $U_{q}(\mathfrak{n})$-invariant elements in $A$.

(ii) $B$ is generated by elements $c^{i}, 1 \leq i \leq m$, and $B=\mathbb{C}\left[c^{m}\right] \ldots\left[c^{2}\right]\left[c^{1}\right]$ is an algebra of functions on a quantum affine space with SQCG $\left\{c^{1}, \ldots, c^{m}\right\}$.

(iii) There exist nonzero elements $c_{\beta}, \beta \in \Delta^{+}$, in $A$ which $q$-commute, $q$-commute with $c^{i}, 1 \leq i \leq m$, and satisfy : $E_{\alpha} \cdot c_{\beta}=\delta_{\alpha \beta} c_{\beta}^{>}, \alpha \leq \beta$, where $c_{\beta}^{>}$is either $c_{\gamma}$, $\gamma>\beta, E_{\beta}$-invariant, or $c^{j}, 1 \leq j \leq m$.

Proposition. Let $A$ be a noetherian domain. If $A$ satisfies the property $(\mathcal{P})$, then Fract $A$ is isomorphic to a quantum Weyl field. To be precise, if $B$ is the algebra of $U_{q}(\mathfrak{n})$-invariant elements in $A$, then Fract $A$ is isomorphic to the skew field of fractions of $B\left[c_{\beta_{N}}\right] \ldots\left[c_{\beta_{2}}\right]\left[c_{\beta_{1}}\right]$.

Proof. Suppose that $A$ satisfies the property $(\mathcal{P})$. For all $\beta=\beta_{i}$ in $\Delta^{+}$, note $S_{i}$ the multiplicative set generated by $s_{i}:=c_{\beta}^{>}$; cf. (iii). We can define the following elements : $a_{\beta}=\left(c_{\beta}^{>}\right)^{-1} c_{\beta} \in$ Fract $A$.

In the context of the previous assertion, we set (improperly) $C_{S}=\bigcup_{p \geq 0} C s^{-p}$, where $S$ is the multiplicative set generated by $s$. Recall that $A$ is a domain. The conditions of the assertion are easily verified from the property $(\mathcal{P})$. We have:

$$
\begin{aligned}
A= & A^{0} \subset A_{S_{1}}^{1}\left[a_{\beta_{1}}\right] \subset A_{S_{2}}^{2}\left[a_{\beta_{2}}\right]\left[s_{1}^{-1}, a_{\beta_{1}}\right] \\
& \subset \ldots \subset A_{S_{N}}^{N}\left[a_{\beta_{N}}\right]\left[s_{N-1}^{-1}, a_{\beta_{N-1}}, \ldots, s_{1}^{-1}, a_{\beta_{1}}\right] .
\end{aligned}
$$


Moreover, from (iii), $c_{\beta_{N}}^{>} \in B$, so $c_{\beta_{N}} \in B\left[a_{\beta_{N}}\right]$. Inductively, we can prove that $B\left[a_{\beta_{N}}\right] \ldots\left[a_{\beta_{1}}\right]$ contains all the $s_{i}$. This and $(*)$ imply that Fract $A$ is isomorphic to the skew field of fractions of $B\left[a_{\beta_{N}}\right] \ldots\left[a_{\beta_{1}}\right]$. The property $(\mathcal{P})$ (iii) asserts that the $a_{\alpha}$ are in the skew field of fractions of $B\left[c_{\beta_{N}}\right] \ldots\left[c_{\beta_{2}}\right]\left[c_{\beta_{1}}\right]$. By the Taylor lemma, these extensions are (right) transcendantal. Our proposition follows.

2.3. Fix $1 \leq l \leq N$ and $\beta=\beta_{l}$. The reduced decomposition of $w_{0}$ being fixed as in 1.3, we define the elements $y_{l}$ of the Weyl group : $y_{0}=I d, y_{l}=s_{i_{1}} s_{i_{2}} \ldots s_{i_{l}}, l>0$. Then, we introduce in $R^{+}: c_{\beta}=c_{y_{l-1} \varpi_{i_{l}}, \varpi_{i_{l}}}^{\varpi_{i}}, c_{\beta}^{>}=c_{\beta} . E_{\beta}$.

Lemma. Let $I_{l}=\left\{p, l<p \leq N \mid i_{p}=i_{l}\right\}$. If $I_{l}$ is empty, $c_{\beta_{l}}^{>}=c_{w_{0} \varpi_{i_{l}}, \varpi_{i_{l}}}^{\varpi_{i_{l}}}$. If not, let $l^{\prime}$ be the minimal element in $I_{l}$; then $c_{\beta_{l}}^{>}=c_{\beta_{l^{\prime}}}$ (up to a multiplicative scalar).

Proof. Fix $l$. Set $j=i_{l}$. We show the second assertion of the lemma; the first is similar. We have $y_{l^{\prime}-1}\left(\varpi_{j}\right)=y_{l-1} s_{i_{l}} \ldots s_{i_{l^{\prime}-1}}\left(\varpi_{j}\right)=y_{l-1} s_{i_{l}}\left(\varpi_{j}\right)=y_{l-1}\left(\varpi_{j}-\alpha_{j}\right)=$ $y_{l-1}\left(\varpi_{j}\right)-\beta$. From Assertion 1.4, with $\lambda=\varpi_{j}$ and $w=y_{l-1}$, it is enough to prove that $v_{\varpi_{i_{l}}}^{*}$. $E_{\varpi_{i_{l}}}=v_{s_{i_{l}} \varpi_{i_{l}}}$. This is clear by the Weyl character formula and we can conclude the lemma.

Proposition. Let $S$ be the multiplicative set generated by the $c_{\alpha}^{>}, \alpha \in \Delta^{+} . S$ is a Ore set in $R^{+}$. Let $a_{\beta}=\left(c_{\beta}^{>}\right)^{-1} c_{\beta} \in S^{-1} R^{+}$. We have $a_{\alpha^{\prime}} a_{\alpha}=q^{\left(\alpha, \alpha^{\prime}\right)} a_{\alpha} a_{\alpha^{\prime}}, \alpha$, $\alpha^{\prime} \in \Delta^{+}, \alpha^{\prime}<\alpha$.

Proof. As in the previous proof, we fix $\beta=\beta_{l}$ in $\Delta^{+}, 1 \leq l \leq N$, and $i_{l}=j$. From Assertion 1.4, the extremal vector $v_{y_{l-1} \varpi_{j}}^{*}$ in $L_{q}\left(\varpi_{j}\right)^{*}$ is annihilated by the right action of $E_{\alpha}, \alpha>\beta$, and $F_{\alpha}, \alpha \leq \beta$. So, this holds for $c_{\beta}$. From (1.5.1) and (1.5.2), we can deduce that the $c_{\beta}, \beta \in \Delta^{+}, q$-commute. By Lemma 2.3 and by 14. Corollary 9.1.4], this is also true for the $c_{\beta}^{>}, \beta \in \Delta^{+}$. The first assertion of the proposition follows from loc. cit., [Lemma A.2.9], and loc. cit., [Lemma 9.1.10]. We used loc. cit., [Proposition 9.1.5] to calculate the exponent of $q$ in the formula.

Remark. The fact that $S$ is a Ore set is not essential for the next section. Indeed, the elements $a_{\beta}$ defined above exist at least in Fract $R^{+}$, because $R^{+}$is a noetherian domain, cf. [14, 9.1.11].

\section{Applications}

3.1. In this section, we give a list of quantum algebras which satisfy the desired property.

Theorem. The skew field of fractions of the algebra $R^{+}$, resp. $S_{w}^{+}$, resp. $U_{q}(\mathfrak{n})$, resp. $U_{q}(\mathfrak{b})$, is isomorphic to a quantum Weyl field of dimension $N+n$, resp. $l(w)+n$, resp. $N$, resp. $N+n$.

Proof. By [14, Chapter 7, Chapter 9], all the algebras in the claim are noetherian domains. Let's verify the assertions of the property $(\mathcal{P})$.

For $R^{+}$, the action of (i) is the right regular action, which is locally finite. The algebra of $U_{q}(\mathfrak{n})$-invariant elements in $R^{+}$is generated by the $c_{w_{0} \varpi_{i}, \varpi_{i}}^{\varpi_{i}}, 1 \leq i \leq n$, which $q$-commute by [14, 9.1.4]. Then, (iii) is given by Lemma 2.3 and Proposition 2.3 .

The assertion for $S_{w}^{+}$is similar. We may consider, without loss of generality, the case where $w=s_{i_{N-l(w)+1}} \ldots s_{i_{N}}$. Set $\beta:=\beta_{N-l(w)+1}$. We prove the theorem with the help of (i) the right action of $U_{q}(\mathfrak{n})$, (ii) the $U_{q}(\mathfrak{n})$-invariant elements $c_{w \varpi_{i}}^{\varpi_{i}}, \varpi_{i}$, 
$1 \leq i \leq n$, (iii) the $q$-commuting elements $c_{\alpha}, \alpha \leq \beta$. Indeed, (i) and (iii) are clear, and (ii) follows from the fact that $\left(V_{w}^{+}(\lambda)^{*}\right)^{U_{q}(\mathfrak{n})}$ is generated by $c_{w \lambda, \lambda}^{\lambda}$.

Let $\varphi$ be the restriction homomorphism from $U_{q}(\mathfrak{g})^{*}$ to $U_{q}\left(\mathfrak{b}^{-}\right)^{*}$ and $J^{-}=$ $K \operatorname{Ker} \varphi \cap R$. We know (cf. 14, 9.1.10, 9.2.11]) that $\varphi$ restricts to an embedding from $R^{+}$to $R / J^{-}$, and this map is surjective up to localization. It is also well known (cf. 3]) that there exists an algebra antihomomorphism $R / J^{-} \simeq U_{q}(\mathfrak{b})$. The theorem being true for $R^{+}$, it is true for $U_{q}(\mathfrak{b})$. Let's consider now the previous isomorphism extent to the skew field of fractions. We remark (cf. [4, Lemme 3.4], [5, I Prop. 4.2]) that the image of $a_{\beta}$ is in Fract $U_{q}(\mathfrak{n})$. Thus, we can conclude by (1.2.1).

As in [15, Corollaire 6], we have the following corollary.

Corollary. Let $P$ minimal primitive ideal of $U_{q}(\mathfrak{g})$. Then Fract $U_{q}(\mathfrak{g}) / P$ is isomorphic to a quantum Weyl field of dimension $2 N$.

3.2. In this section section, we make more explicit the system of $q$-commuting generators (SQCG) of Fract $U_{q}(\mathfrak{n})$ for the classical simple Lie algebras $\mathfrak{g}$. Recall that these generators are, from the proof of Theorem 3.1, the images of the elements $c_{\beta}$, $\beta \in \mathcal{R}^{+}$, by the Drinfeld (anti)homomorphism, followed by the natural projection on $U_{q}(\mathfrak{n})$.

We know [6] that there is a natural embedding from $L_{q}(\lambda)^{*}$ to $U_{q}(\mathfrak{n})$ which maps the lowest weight vector of $L_{q}(\lambda)^{*}$ to 1 . It maps the highest weight vector to an element $e_{\lambda}$ of the $q$-center of $U_{q}(\mathfrak{n})$; cf. [7]. An element of $U_{q}(\mathfrak{n})$ will be called almost maximal, and be noted $e_{\lambda}^{i}$, if it is the image of a vector $v_{-s_{i} w_{0} \lambda} \in L_{q}(\lambda)^{*}$, $1 \leq i \leq n, \lambda \in P^{+}$. Remark that those elements can be explicitly computed with the help of [6] Lemme 3.3].

With the standard notations of [2] Planches I à IV], we recall the canonical embeddings of Dynkin diagrams:

$A_{1} \subset \ldots \subset A_{n}, A_{1} \subset B_{2} \subset \ldots \subset B_{n}, A_{1} \subset C_{2} \subset \ldots \subset C_{n}, A_{3} \subset D_{4} \subset \ldots \subset D_{n}$.

If $X$ is a Dynkin label for a classical simple Lie algebra, we denote by $X^{-}$the previous label for the embedding sequence above. We can inductively define the reduced decomposition of the longest element of the Weyl group $w_{0}(X)$ for the Lie algebra of type $X$ by :

$$
w_{0}(X)=w_{0}\left(X^{-}\right) \cdot \begin{cases}s_{n} \ldots s_{2} s_{1}, & \text { if } X=A_{n}, \\ s_{1} \ldots s_{n-1} s_{n} s_{n-1} \ldots s_{1} & \text { if } X=B_{n} \text { or } C_{n}, \\ s_{1} \ldots s_{n-2} s_{n} s_{n-1} \ldots s_{1} & \text { if } X=D_{n} .\end{cases}
$$

This decomposition of $w_{0}$ permits us to obtain inductively our SQCG.

Theorem. The system of $q$-commuting generators corresponding to the simple classical Lie algebra $\mathfrak{g}$ of type $X$ is inductively given by

$$
S Q C G(X)=S Q C G\left(X^{-}\right) \cup \bigcup_{i=1}^{n} e_{\varpi_{i}} \cup \begin{cases}\varnothing & \text { if } X=A_{n}, \\ \bigcup_{i=1}^{n-1} e_{\varpi_{i}}^{i} & \text { if } X=B_{n} \text { or } C_{n}, \\ \bigcup_{i=1}^{n-2} e_{\varpi_{i}}^{i} & \text { if } X=D_{n} .\end{cases}
$$

Remark. This theorem is a generalization of [1, Théorème 2.15] for the classical Lie algebras. Note that, except for $e_{\varpi_{n}}$ if $\mathfrak{g}$ has type $B_{n}, C_{n}, D_{n}$ and $e_{\varpi_{n-1}}$, if $\mathfrak{g}$ has type $D_{n}$, all those elements can be obtained as quantum determinants of a "basic" 
matrix. This can be obtained as in [6] (see also [14, 7.5.5]) by considering exterior powers of $L_{q}\left(\varpi_{1}\right)$.

3.3. Now, we give the proof of a similar theorem for the algebra $R=\mathbb{C}_{q}[G]$. For all $i, j, 1 \leq i, j \leq N$, we denote by $R^{i, j}$ the subalgebra of elements in $R$ which are invariant for the right action of $U_{q}\left(\mathfrak{n}_{\beta_{j}}\right)$ and for the left action of $U_{q}\left(\mathfrak{n}_{\beta_{i}}\right)$ (recall that these actions commute). Set $B=R^{N, N}$.

Fix $\beta=\beta_{l}$. We define the following elements in $C(\rho)$ :

$$
d_{\beta}=c_{y_{l-1} \rho,-y_{l} \rho}, \quad d_{\beta}^{\prime}=c_{y_{l-1} \rho,-y_{l-1} \rho} .
$$

By Assertion 1.4, we prove that $d_{\beta}$ is invariant for the left action of $E_{\alpha}, \alpha \leq \beta$, and of $F_{\alpha}, \alpha>\beta$. Moreover, $d_{\beta}$ is invariant for the right action of $E_{\alpha}, \alpha<\beta$, and of $F_{\alpha}, \alpha \geq \beta$. In the same way, $d_{\beta}^{\prime}$ is invariant for the left and right action of $E_{\alpha}, \alpha<\beta$, and of $F_{\alpha}, \alpha \geq \beta$. Hence, by (1.5.1) and (1.5.2), the elements $d_{\alpha}$ and $d_{\alpha}^{\prime}, \alpha \in \Delta^{+}, q$-commute. We have clearly $d_{\beta} \in R^{l, l-1}$ and $d_{\beta} \in R^{l-1, l-1}$. Moreover, up to a nonzero multiplicative scalar, $E_{\beta} \cdot d_{\beta}^{\prime}=d_{\beta}$ and $d_{\beta} \cdot E_{\beta}=d_{\beta_{l+1}}^{\prime}$, $l \neq N, d_{\beta} . E_{\beta}=c_{w_{0} \rho, \rho} \in B, l=N . \mathbb{C}_{q}[G]$ being noetherian [14, 9.2.2], we can easily modify the proof of Proposition 2.2 to obtain:

Theorem. Fract $\mathbb{C}_{q}[G]$ is isomorphic to a quantum Weyl field of dimension $2 N+n$. To be more precise, Fract $\mathbb{C}_{q}[G]$ is isomorphic to the skew Weyl field of fractions of $B\left[d_{\beta_{N}}\right]\left[d_{\beta_{N}}^{\prime}\right] \ldots\left[d_{\beta_{2}}\right]\left[d_{\beta_{2}}^{\prime}\right]\left[d_{\beta_{1}}\right]\left[d_{\beta_{1}}^{\prime}\right]$.

The generators of $B$, the $d_{\beta}$ and $d_{\beta}^{\prime}$, can be easily expressed as a product of elements of $C\left(\varpi_{i}\right), 1 \leq i \leq n$. Consider the algebra $\mathbb{C}_{q}\left[S L_{2}\right]$ generated by the elements of the quantum matrix : $\left(\begin{array}{ll}a & c \\ b & d\end{array}\right)$. Then the system of $q$-commuting generators provided by the theorem is $\{b, a, c\}$.

\section{REFERENCES}

[1] J. ALEV and F. DUMAS. Sur le corps des fractions de certaines algèbres quantiques, J. Algebra, 170, (1994), 229-265. MR 96c:16033

[2] N. BOURBAKI. Groupes et Algèbres de Lie, Chap. VI, Masson, Paris, 1981.

[3] N. BURROUGHS. Relating the approaches to quantized algebras and quantum groups, Comm. Math. Phys., 133, (1990), 91-117. MR 92c:17018

[4] P. CALDERO. Générateurs du centre de $U_{q}(s l(N+1))$, Bull. Sci. Math., 118, (1994), 177-208. MR 95k:17018

[5] P. CALDERO. Algèbres enveloppantes quantifiées, action adjointe et représentations, Thèse Université Paris VI, (1993).

[6] P. CALDERO. Sur le centre de $U_{q}(\mathfrak{n})$, Beiträge zur Algebra und Geometrie, 35, (1994), 13-23. MR 95d:17009

[7] P. CALDERO. Etude des q-commutations dans l'algèbre $U_{q}(\mathfrak{n})$, J. Algebra, 178, (1995), 444457. MR 96k:17019

[8] P. CALDERO. Invariants in the enveloping algebra of a semi-simple Lie algebra for the adjoint action of a nilpotent Lie subalgebra, Comm. Math. Phys. 189 (1997), 699-707. CMP 98:04

[9] P. CALDERO. On the q-commutations in $U_{q}(\mathfrak{n})$ at roots of one, to appear in J. Algebra.

[10] C. DE CONCINI and V. G. KAC. Representations of quantum groups at roots of 1 , Colloque Dixmier, Progress in Math., 92, (1990), 471-506. MR 92g:17012

[11] V. G. DRINFELD. On almost cocommutative Hopf algebras, Leningrad Math. J., Vol. I, (1990), $\mathrm{n}^{\circ}$ 2, 321-342. MR 91b:16046

[12] A. JOSEPH. A generalization of the Gelfand-Kirillov conjecture, Amer. J. Math., 99, (1977), 1151-1165. MR 57:391 
[13] A. JOSEPH. A preparation theorem for the prime sprectrum of a semi-simple Lie algebra, 48, (1977), 241-289. MR 56:12082

[14] A. JOSEPH. Quantum groups and their primitive ideals, Springer-Verlag, 29, (1995). MR 96d:17015

[15] A. JOSEPH. Sur une conjecture de Feigin, C.R.Acad.Sci., 320, Serie I, (1995), 1441-1444. MR 96f: 17020

[16] V. LAKSHMIBAI, N. RESHETIKHIN. Quantum flag and Schubert schemes, Contemp. Math., 134, (1992), 145-181. MR 94a:14055

[17] S.Z. LEVENDORSKII, Y.S. SOIBELMAN. Some applications of quantum Weyl group, J. Geom. Phys., 7, (1990), 241-254. MR 92g:17016

[18] G. LUSZTIG. Quantum groups at roots of 1, Geom. Ded., 35 (1990), 1-25. MR 91j:17018

[19] F. MILLET-FAUQUANT, Sur une algebre parabolique $P$ de $U_{q}(s l(N+1))$ et ses semiinvariants par l'action adjointe de $P$, preprint.

[20] Y. NOUAZÉ, P. GABRIEL. Idéaux premier de l'algèbre enveloppante d'une algèbre de Lie nilpotente, J. Algebra, 6, 77-99, (1967). MR 34:5889

[21] A.N. PANOV. The skew field of rational functions on $G L_{q}(n, K)$, Transl. from Funk. Anal., Vol. $28, \mathrm{n}^{0}$ 2, p. $75-77$, 1994. CMP 94:14

[22] C.M. RINGEL. Hall algebras and quantum groups, Invent. Math., 101, (1990), 583-592. MR 91i:16024

Institut Girard Desargues, UPRS-A-5028, Université Claude Bernard lyon I, Bat 101, 69622 Villeurbanne Cedex, France

E-mail address: caldero@desargues.univ-lyon1.fr 